ISSN: 0213-2079 - ISSN electrónico: 2386-3889

DOI: https://doi.org/10.14201/shhmo2020422197217

\title{
LOS SITIOS REALES COMO ELEMENTOS CLAVE DE LAS MONARQUÍAS EUROPEAS DE LA EDAD MODERNA: UNA APROXIMACIÓN
}

\section{The Royal Sites as Key Elements of the Early Modern European Monarchies: An Approach}

José Eloy HORTAL MUÑOZ

joseeloy.hortal@urjc.es

Universidad Rey Juan Carlos

Fecha de recepción: 12/02/2020

Fecha de aceptación: 20/09/2020

RESUMEN: A lo largo del tiempo, los Sitios Reales europeos han sido profusamente estudiados desde varios puntos de vista. Sin embargo, dichos estudios no siempre han tenido en cuenta los diversos elementos que componían los Sitios Reales en la Edad Moderna, pues mayoritariamente se han centrado en los edificios y en sus colecciones artísticas y patrimonio, prestando en ocasiones algo de atención a la caza o el urbanismo. Esto ha provocado que numerosos y cruciales componentes de los Sitios Reales hayan sido olvidados, y no se haya tenido en cuenta que dichos lugares deben estudiarse desde un punto de vista global para su correcta comprensión. Gracias a los avances metodológicos de los últimos años, están apareciendo trabajos sobre dichos lugares desde nuevas perspectivas que nos ayudan a comprender mejor su verdadero significado durante el periodo. El presente artículo pretende ofrecer una aproximación sobre los avances que nos encontramos en la actualidad sobre el estudio de los Sitios Reales, así como el papel que jugaron en la configuración de las monarquías europeas de la Edad Moderna.

Palabras clave: Sitios Reales; Europa; Edad Moderna.

Ediciones Universidad de Salamanca / @®@@ Stud. his., H. ${ }^{a}$ mod., 42, n. 2 (2020), pp. 197-217 
JOSÉ ELOY HORTAL MUÑOZ

LOS SITIOS REALES COMO ELEMENTOS CLAVE DE LAS MONARQUÍAS EUROPEAS

DE LA EDAD MODERNA: UNA APROXIMACIÓN

ABSTRACT: All over history, european Royal Sites have been deeply studied from different approaches. Nonetheless, those studies not always had taken into account every elements that composed these Royal Sites at Early Modern Times, as they mainly focused on buildings, artistic collections and heritage, paying some attention to royal hunt or urbanism. In this sense, numerous and crucial elements of these Royal Sites have been neglected, while we know that these places must be studied from a holistic point of view for its better comprenhension. Thanks to the methodological improvements that social sciences have been doing in lasts years, lately studies with new perspectives are appearing, which allows us to better comprenhend the true meaning of the Royal Sites during the period. This article intends to bring an approach on the advances we have nowadays over the study of the Royal Sites, as well as the role they played in the shape of the early modern european monarchies.

Keywords: Royal Sites; Europe; Early Modern era.

\section{LOS SITIOS REALES DURANTE LA EDAD MEDIA Y MODERNA: DE LA AUSENCIA DE PLAN A LA ESTRUCTURACIÓN DEL TERRITORIO}

Hoy en día, los Sitios Reales de los diversos países europeos son vistos en muchos casos como residencias de las familias reales que vivían aisladas de la sociedad, y en donde los monarcas y sus familias únicamente se dedicaban a «holgar» $\mathrm{y}$ «solazarse», tal y como se decía en la época, cazando o realizando fiestas. Sin duda, esa imagen es en esencia la que evocaron historiadores y literatos durante el siglo XIX, idea que debemos desterrar. Debido a ello, la mayoría de los estudios que se han llevado a cabo sobre estos lugares se han centrado únicamente en los edificios y en sus colecciones artísticas, prestando en ocasiones algo de atención a la caza o el urbanismo ${ }^{1}$. Esto ha provocado que numerosos y cruciales componentes de los Sitios Reales hayan sido olvidados, y no se haya tenido en cuenta que dichos lugares deben estudiarse desde un punto de vista global para su correcta comprensión.

Por lo tanto, y en primer lugar, se hace necesario acotar qué entendemos por Sitio Real. En algunos países como España o Italia, dicho término no supone ningún problema conceptual, pues se sigue utilizando en el lenguaje común -aunque en la mayoría de las ocasiones el imaginario colectivo remite únicamente a las

1. Con algunas notables excepciones, como Maroteaux, V. (2000). Versailles. Le Roi et son Domaine. París: Editions Picard. 
JOSÉ ELOY HORTAL MUÑOZ

LOS SITIOS REALES COMO ELEMENTOS CLAVE DE LAS MONARQUÍAS EUROPEAS

DE LA EDAD MODERNA: UNA APROXIMACIÓN

construcciones arquitectónicas- e, incluso, las entidades encargadas de gestionar dichos lugares siguen empleando esa expresión. Sin embargo, en otros lugares como Reino Unido no se suele emplear, y el término Royal Site evoca emplazamientos arqueológicos relacionados con la Edad Antigua ${ }^{2}$, partiendo del concepto de Site expresado por Hilda Kuper en los años 70 del siglo pasado ${ }^{3}$. Por lo tanto, el término más empleado suele ser Residence, o Court Residence, al igual que sucede en otros ámbitos como el germánico, con su equivalente Residenz ${ }^{4}$. Más ajustado nos parece el término Domaine que se emplea en Francia y en otros lugares cómo en los Países Bajos, Domein, pues indica todo lo comprendido en un territorio, en este caso de posesión del Soberano.

Debido a esta falta de precisión, desde hace varios años llevamos trabajando en una definición que sirva para demostrar qué entendemos conceptualmente por Sitio Real, con el fin de poder extenderla a aquellos ámbitos historiográficos que no la incluyen en su acepción global 5 . En virtud de ello, defendemos que en la Edad Moderna el término «Sitio Real» se aplicaba a las propiedades de las dinas-

2. Entre los que resultan famosos los «six Royal Sites» de la Irlanda antigua, que se explican en Newman, C. (1998). Reflections on the making of a 'royal site' in early Ireland, World Archaeology, 30(1), 127-141, https://doi.org/10.1080/00438243.1998.9980401.

3. Definición que podemos encontrar en su artículo Kuper, H. (1972). The Language of Sites in the Politics of Space, American Anthropologis, 74, 411-425, «a particular piece of social space, a place socially and ideologically demarcate and separate». En los últimos años se ha ido más allá y, según Dillon, J. (2010). The language of space in court performance, 1440-1625. Cambridge: Cambridge University Press, 9, estos espacios físicamente separados del resto, como fue el caso de los Sitios Reales, generaban y potenciaban siempre el sentido de autoridad o sacralidad.

4. El debate sobre el término tiene luenga tradición, tal y cómo podemos ver en Engel, E. y Lambrecht, K. (1995). Hauptstadt-Residenz-Residenzstadt-Metropole-zentraler Ort. Probleme ihrer Definition und Charakterisierung. En E. Engel, K. Lambrecht y H. Nogossek (eds.), Metropolen im Wandel. Zentralität in Ostmitteleuropa an der Wende vom Mittelalter zur Neuzeit. Berlín: De Gruyter Akademie Forschung (= Forschungen zur Geschichte und Kultur des ostlichen Mitteleuropa), 11-31. Al estudio de dicho término de Residence de un modo transdisciplinar y transnacional se refirieron los trabajos de la red PALATIUM, Research Networking Programme de la European Science Foundation dirigida por Krista de Jonge. Dicho proyecto, cuyos trabajos se llevaron a cabo entre 2010 y 2015, tenía como título completo el de Court Residences as Places of Exchange in Late Medieval and Early Modern Europe (14001700). Sus actividades y publicaciones pueden consultarse en http://www.courtresidences.eu/ index.php/home/. Esta iniciativa va a tener su continuidad en la recién iniciada European Training Network PALAMUSTO (Research and Training for the Palace Museum for Tomorrow), también dirigida por Krista de Jonge.

5. Además de a nivel académico, en la URJC estamos mostrando dicha definición y concepto a través de diversas iniciativas de diseminación vinculadas a la valorización del patrimonio, el turismo, la arquitectura, las TIC o la educación; iniciativas todas que convergen en el MOOC Los Sitios Reales como recurso profesional, que se encuentra alojado en la plataforma URJCx y del cuál se han celebrado ya cuatro ediciones (link: https://urjcx.urjc.es/courses/

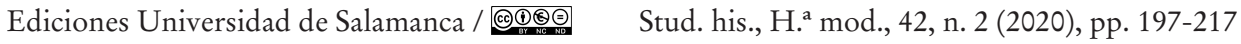


JOSÉ ELOY HORTAL MUÑOZ

LOS SITIOS REALES COMO ELEMENTOS CLAVE DE LAS MONARQUÍAS EUROPEAS

DE LA EDAD MODERNA: UNA APROXIMACIÓN

tías reinantes, donde el Soberano u otros miembros de la dinastía vivían, habían vivido o existía la expectativa de que podrían residir en algún momento, tanto de modo permanente como estacional. En este sentido, los Sitios Reales no se referían únicamente a los palacios, pues en dichos lugares nos encontramos también con bosques, jardines, espacios agrícolas, fábricas y centros urbanos. Por supuesto, en ellos debemos incluir también todos aquellos monasterios reales o conventos fundados o patrocinados por la familia real, que podían tenían un cuarto real o un panteón real adosado, por lo que estaban íntimamente ligados a la dinastía. Del mismo modo, miembros de dicha dinastía, generalmente mujeres, solían profesar en ellos; este fue el caso, por ejemplo, de los monasterios de San Lorenzo de El Escorial, Westminster, Val-de-Grâce o Mafra.

Aunque ya existían desde la Edad Antigua residencias reales e, incluso, los soberanos llevaban a cabo movimientos estacionales entre ellas ${ }^{6}$, debemos considerar que el concepto de Sitio Real no adquiriría su verdadero significado hasta la Baja Edad Media. En este periodo, los monarcas de los diversos reinos europeos fueron utilizando, construyendo y reformando diferentes edificios a lo largo de la geografía de sus territorios, con el fin de que les sirvieran de estancia, y con ellos a sus cortes ambulantes, reconociéndose la propiedad sobre ellos y llevándose a cabo numerosas obras para consolidar las nuevas entidades territoriales que se iban gestando. Poco a poco, estas redes de Sitios Reales se convertirían en fundamentales en la consolidación del poder del Soberano y en la promoción de su soberanía, al tiempo que ayudaban al control de enormes posesiones de terreno a través de las Jornadas que realizaban los monarcas a dichos lugares. Algunos de estos lugares tuvieron su origen como cotos de caza, actividad que era más que un mero entretenimiento, pues tenía propósitos políticos y de representación, ya que durante dicha actividad el Soberano se encontraba con los miembros más relevantes de su sociedad. Del mismo modo, significaba una actividad física y de entrenamiento para la guerra tanto para los diversos nobles como para la familia real, siempre teniendo en cuenta que desde el siglo XVI la misma fue abandonando los campos de batalla (Anthenaise y Chatenet, 2007; Allsen, 2009; Merlotti, 2017). A estos cotos de caza, se añadirían posteriormente centros urbanos.

course-v1:URJCx+URJCx110+AHx05/about). Este curso fue finalista de los premios Medea Awards 2019 en la categoría de Professionally Produced Educational Media.

6. Este es el caso, por ejemplo, de las residencias de los aqueménidas persas de Babilonia, Ecbatana, Pasargada, Persépolis y Susa, que estaban conectadas entre sí formando un cuadrilátero. A ellas se desplazaba anualmente el soberano persa acompañado de un gran cortejo, nomadismo que inició Ciro II (tal y cómo explica Velázquez Muñoz, J. (2011). El Gran Rey en movimiento. Banquetes y partetaš. Espacio, Tiempo y Forma. Serie II, Historia Antigua, 24, 161-188).

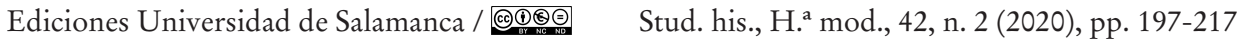


JOSÉ ELOY HORTAL MUÑOZ

Durante los primeros siglos, estos lugares se reformaban mediante organizaciones creadas ad-hoc, compuestas por arquitectos, oficiales y secretarios nombrados ex profeso para una tarea concreta. Sin embargo, ya en el siglo XIV, y en especial a partir de 1370, surgieron instituciones en las cortes del Norte de Europa encaminadas a centralizar la construcción y el diseño de dichos lugares ${ }^{7}$. Los primeros reinos interesados en potenciar dichas instituciones fueron los principales implicados en la Guerra de los Cien Años, apareciendo la primera institución relevante conocida en Inglaterra, con la creación en 1378 de The King's Works, que estaría compuesta por un clerk ayudado por un comptroller, un chief mason y un chief carpenter, cuya labor estaría principalmente centrada en Londres y alrededores (Colvin, 1963-1982, vol. I; Harvey, 1941). Una década después, en concreto en 1388, se crearía en Francia la figura del visiteur general, y se nombró a un maistre maçon des oenvres $d u$ roi par tout son royaume, el cual, pese al nombre, solo centraría su labor en París y alrededores (Lardin, 1999; Chapelot, 2001). Ambas instituciones se convirtieron en modelo y se expandieron a otros lugares de Europa, comenzando por los príncipes de la sangre franceses como el Duque de Berry o el de Borgoña, creándose en dicho ducado las Ouvrages de la Cour en 1395 (Jacquier, 2001). Posteriormente, se expandirían a los territorios germánicos (Bürger, 2009) y, finalmente, a los mediterráneos, como fue el caso de Saboya (Poisson, 2001), o de Castilla con los Reyes Católicos (Domínguez Casas, 1993). Estas instituciones serían las responsables de gestionar, organizar y planificar los edificios, sitios y espacios reales hasta el final del Antiguo Régimen, aunque sufrirían diversos cambios en los siglos siguientes, pues tuvieron que adaptarse a la naturaleza cambiante de las diversas monarquías, así como al ritmo y decurso vital de cada una de ellas.

En efecto, a lo largo de la Edad Moderna los diversos soberanos expandieron dichas administraciones, al ser conscientes del potencial político, social y cultural de dichos lugares. En virtud de ello, en el siglo XVI las monarquías más relevantes fueron un paso más allá, al generar una política arquitectónica conjunta para todo el reino, estableciendo cuerpos permanentes encargados de la gestión de toda esa red de propiedades para extender la presencia del soberano a todos los rincones

7. Las instituciones que gestionaban estos Sitios Reales han sido estudiadas hasta ahora en la mayoría de las ocasiones desde un punto de vista institucional y nacional, por lo que no se ha comparado de forma sistemática lo que sucedía en unos reinos y otros. Esta es una carencia que se ha pretendido subsanar en el Simposium Building the Presence of the Prince: the institutions related with the ruler's works as key elements of the European courts (XIVth-XVIIth centuries), dirigido por los profesores Merlijn Hurx y José Eloy Hortal Muñoz, y celebrado en Utrecht (Países Bajos) los días 8 y 9 de noviembre de 2019. En dicho encuentro se estudiaron y compararon sistemáticamente dichas instituciones desde el siglo XIV hasta el XVII de un modo transdisciplinar y transnacional, tratando de identificar sus características locales y unas europeas comunes, estudiando su naturaleza, su propósito y su estructura organizativa. Los resultados serán publicados en un volumen en la editorial Brepols en 2021.

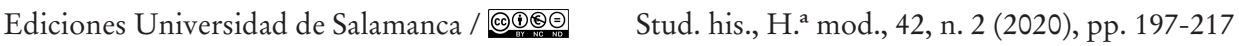


JOSÉ ELOY HORTAL MUÑOZ

LOS SITIOS REALES COMO ELEMENTOS CLAVE DE LAS MONARQUÍAS EUROPEAS

DE LA EDAD MODERNA: UNA APROXIMACIÓN

del territorio. Monarcas como Enrique VII, con la Office of the King's Works (Colvin, 1963-1982, vols. IV-V), Felipe II con la Junta de Obras y Bosques (García Morales, 1990; Díaz González, 2002; Martínez Millán, 2017), o Francisco I con la surintendance des bâtiments (Nan Rosenfeld, 1977; Barbiche, 1984; Sarmant, 2003), aprovecharían dichas instituciones para transmitir a todo el reino a través de ellas la imagen exacta de su soberanía que querían mostrar.

Finalmente, durante el siglo XVII, los Sitios Reales y las instituciones que los gestionaban se utilizarían para poder afrontar los diversos desafíos que las monarquías europeas tuvieron durante dicho periodo, ya como verdaderos centros de poder. En especial, se estrecharían los vínculos entre los Sitios Reales y la capital, uniendo efectivamente Corte, Casa y Territorio ${ }^{8}$.

Esto fue posible gracias a la creación de nuevos tipos de oficios en dichos Sitios Reales, con el consiguiente aumento del número de trabajadores y moradores en dichos lugares, y de una mejor condición social que antaño, pues tenían como fin poder dar el salto a la Corte; cuestión que sería posible cuando los validos o ministros principales de cada monarquía se pusieron al frente de dichos lugares. Conscientes del potencial de dichos Sitios Reales para integrar de forma más efectiva a unos reinos sacudidos por las numerosas guerras y revueltas del periodo, así como para poder controlar mejor el entorno del rey, personajes como Olivares en la Monarquía Hispana ${ }^{9}$, el Cardenal Mazarino en Francia ${ }^{10}$, o el duque de Buckingham en Inglaterra, se pondrían al frente de dichos lugares directa o indirectamente, a través de «hechuras».

8. Tal y como se estudia en detalle para Castilla y Flandes en Hortal Muñoz, J. E. (2017). La unión de la Corte, la Casa y el territorio en la Monarquía Hispana de los siglos XVI y XVII: las guardas reales y los Sitios Reales. Revista Escuela de Historia, 16(1). En el mismo, se analiza en profundidad el caso de las guardas reales, pero hay otros ejemplos vinculados a la caza, capilla, particulares,...

9. El conde-duque de Olivares fue alcaide de los Reales Alcázares de Sevilla, del cuarto real de San Jerónimo, que derivaría después en el Sitio Real del Retiro, de Vaciamadrid y de la Zarzuela. Ya Lerma había intuido el potencial de estos lugares, pero serían Olivares y sus sucesores, como don Luis de Haro, el marqués de Eliche o el conde de Monterrey, quienes explotarían convenientemente el potencial de estos lugares, reforzando la vinculación de los mismos con la Corte, la cual fue mucho más profunda a partir de Felipe IV (Hortal Muñoz, J. E. (2014). La integración de los Sitios Reales en el sistema de Corte durante el reinado de Felipe IV, Libros de la Corte, 8, 27-47).

10. En Archives Nationales de France [en adelante ANF], O1, 2387, s. f., vemos en el «Estat des gaiges et appointements des officiers» de Fontainebleau en 1646, como aparece «Monsieur le Cardinal Mazarine capitaine concierge, surintendant et ordonnative dudict bastiment dudict chateau de fontainebleau pour ses gaiges intitule la Somme de 6100 livres», así como en años sucesivos. Posteriormente, en Francia se daría un paso más allá al encontrarnos a Colbert como surintendant et ordonnateur generale des batiments de Sa Maiesté.

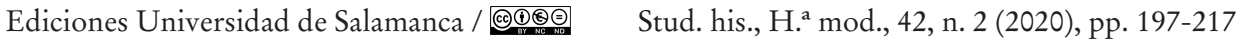


Gracias a ello, los Sitios Reales se acabaron convirtiendo en verdaderos centros de poder que ayudaron a configurar las monarquías modernas europeas. Esta cuestión se puede encontrar ya en los discursos de la Edad Media y de la Edad Moderna que dieron forma y justificaron el poder real. En concreto, en la tradición de los espejos de príncipes, que desde la Baja Edad Media recuperaron y adaptaron la idea clásica de la Oeconomica, o doctrina que explica el gobierno sobre la Casa y en la que los reyes eran representados como Pater Familias, gobernando sobre la Monarquía como un padre sobre su familia y en donde la Casa Real constituía el centro del poder político (Lambertini: 1984; Frigo: 1985; Brunner: 2010). Según esta doctrina, la familia real no se restringía únicamente a los reyes y a sus familiares, si no que incluía a los servidores que trabajaban en las Casas Reales escogidos entre los leales al monarca. De este modo, la familia del rey incluía miembros de las más importantes familias nobiliarias, que al fin y a la postre estaban organizados como familias «extensas», con ramificaciones hasta el poder local a través de «brokers». De este modo, como cabeza de la familia real, el poder de los reyes se extendía a todos los confines del reino, más allá del control directo que tenía sobre los dominios reales.

Central en la idea de la Oeconomica fue el concepto de virtud. Como cabeza de la casa «extensa», el Pater Familias era responsable, no solo, del abastecimiento material de la Casa, sino también de gobernar la misma con autoridad moral, lo que se expresaba en primera instancia en una justa distribución de los beneficios económicos. En una escala más amplia, estas virtudes se expresaban a nivel político, constituyendo la Casa el centro del dominio político sobre la Monarquía (Hortal y Versteegen, 2016: caps. 1 y 2).

\section{ESPACIO, CORTE, SOCIEDAD Y CULTURA}

Los Sitios Reales, por tanto, fueron la materialización de esta idea, lo que explica que estos complejos lugares no solo incluyeran los palacios y sus jardines y cotos de caza, si no también territorios agrícolas, fábricas o centros urbanos, que tenían una función de abastecimiento material de la Casa, al tiempo que también representaban la autoridad del poder real. Esto explica que dichas casas fueran no solo las más espléndidas del reino, sino también las de organización más compleja, desde un doble punto de vista.

En primer lugar, la Casa Real no se concentraba únicamente en un palacio, si no en una red de lugares que albergaban a la familia real en diferentes periodos del año. Cada uno de estos lugares, que solían situarse en los cuatro puntos cardinales, tenía sus propias características morfológicas y de configuración del territorio, por lo que cumplían funciones diferentes en la organización económica y abastecimiento material de la Casa. Además, mediante esta red, el monarca extendía su poder político directo sobre territorios más amplios. 
JOSÉ ELOY HORTAL MUÑOZ

LOS SITIOS REALES COMO ELEMENTOS CLAVE DE LAS MONARQUÍAS EUROPEAS

DE LA EDAD MODERNA: UNA APROXIMACIÓN

En segundo lugar, los diversos miembros de la dinastía real necesariamente tuvieron que distinguirse del resto de familias nobles, por lo que organizaron sus casas a mayor escala, uniéndose sus palacios y territorios anexos a dicha constelación de Sitios Reales.

En efecto, una vez dichos monarcas decidieron fijar capitales en sus reinos, dieron inicio a un sistema de movimiento estacional y de rotación de sus cortes (Sancho y Martínez, 2003; Merlotti, 2016). En un principio, este movimiento sería irregular y muy vinculado a los gustos y personalidad de cada monarca, pero a lo largo de la Edad Moderna se iría fijando de manera progresiva. Este movimiento establecía que en los meses de invierno el monarca y su entorno tenían su residencia en el palacio principal de la capital, para viajar en los meses de clima más benigno a pabellones de caza o lugares de recreo, incrementándose así la relevancia e impacto de dichos lugares sobre el territorio. En este sentido, una constelación de tres círculos concéntricos de Sitios Reales diferentes se fue gestando, organizado en diferentes sistemas dependiendo de cada Monarquía.

El primer círculo sería el del Sitio Real principal, que solía albergar la Casa y Corte del Soberano en esos meses de invierno, aunque no solo. En efecto, estas constituían únicamente el núcleo de un entorno más amplio que incluía reinas, herederos, príncipes, reinas viudas, infantas u otros miembros de la familia real; los cuáles solían tener su propia Casa y, en algunos casos, su propia Corte, formando una constelación de casas dinásticas (Vermeir, Raeymaekers y Hortal, 2014: 7-20). Estas residencias solían estar localizadas en la capital, pero dependía de cada Monarquía.

En Austria, por ejemplo, desde la Edad Media el Hofburg había sido la residencia más importante de los Habsburgo (Karner, 2014; Kurdiovsky et alii, 2015), pero no la única, pues había residencias en Innsbruck y Gratz, así como Wiener Neustadt y Linz. Sería, sin embargo, Fernando I el creador de un verdadero sistema de Sitios Reales, pues desde que subió al trono en 1521 procuró generar ese sistema donde se pudieran cumplir los nuevos requerimientos económicos, sociales y defensivos, así como ceremoniales y representativos del arte del Renacimiento, de sus territorios $^{11}$. La base del sistema serían las ya indicadas residencias tradicionales heredadas de Maximiliano I, a las que se incorporarían en 1526, tras acceder a la corona de Bohemia, las de Praga y otros centros como Brno, Budysjin/Bartzen y Wroclaw. En Hungría, por su parte, se incorporarían Buda y Pressburgo, aunque en pugna

11. Para la configuración del sistema de Sitios Reales del Sacro Imperio con Fernando I, Hausenblasova, J. (2017). A New Monarch and a New System of Residences: Ferdinand I Habsburg as the Founder of the Network of Main and Occasional Residences in the Habsburg Empire. En S. Dobalova e I. P. Muchka (eds.), Looking for Leisure. Court Residences and their Satellites, 1400-1700. Praga: Palatium, 46-61. Una breve descripción de la evolución del mismo en Jeitler, M. (2017). Hunt, Amusement and Representation: The Viennese Hofburg and Its 'Satellites' in the Seventeenth Century, en el mismo volumen, 274-276.

Ediciones Universidad de Salamanca / @®@@ Stud. his., H. ${ }^{a}$ mod., 42, n. 2 (2020), pp. 197-217 
constante con los turcos por dichos lugares, y en la década de 1530 llevó a cabo un plan completo de remodelación de dichos espacios con influencias de las cortes hispana y de los Países Bajos. Después de 1540 se crearían más Sitios Reales -caso de Podebrady, Krivoklat, Kolin y Tachov-, así como en una nueva fase a partir de 1547. Fernando no pudo finalizar completamente el sistema y Maximiliano II continuó la tarea, que se vería entorpecida cuando el papel de Viena como centro de la Corte se vio afectado en el momento en que Rodolfo II decidió trasladar la capital a Praga desde 1582 a 1612. Tras la muerte del soberano, Viena recobró su posición con el Hofburg como palacio principal, cesando Innsbruck y Graz de ser la residencia de dos ramas menores de la familia Habsburgo, tal y cómo habían sido antaño. Cuando la corte imperial se reestableció en Viena, Praga, Presburgo, Buda, Graz, Innsbruck, Regensburg, Augsburgo y Frankfurt, junto con sus respectivos Sitios Reales, volvieron a formar parte del itinerario regular de las Jornadas de los Habsburgo y sus visitas reales (Duindam, 2003: 145-149).

Por su parte, la monarquía francesa siempre tuvo París como capital, con un notable impacto en la configuración de la ciudad (Bove, Gaude-Ferragu y Michon, 2017), siendo las residencias de Louvre y Tullerías las principales. Sin embargo, los reyes franceses alternarían sus estancias allí con otros lugares, caso de los châteaux de Chambord, Madrid y otros alrededor del río Loira (principalmente durante el siglo XVI) y domaines como Saint-Germain-en-Laye, Fontainebleau o Compiègne. Como es bien sabido, Luis XIV decidió en 1682 que Versalles, que había sido edificado en 1623 por su padre como lugar de caza, se convirtiera en el principal Sitio Rea ${ }^{12}$. Con el fin de organizar dicho sistema, Francisco I creó la surintendance des bâtiments, institución que sería perfeccionada por Enrique IV.

Por otro lado, la monarquía inglesa siempre centró su presencia en Londres, con Westminster como su residencia real principal hasta que en 1700 pasó a serlo St. James's Palace, pero dotando de sentido simbólico otros Sitios Reales en Inglaterra (Greenwich, Eltham, Hampton Court,...), Gales (Caernavon), Escocia (Balmoral) e Irlanda (Dublín). La «edad de oro» de los Sitios Reales ingleses tendría lugar durante el reinado de Enrique VIII (1509-1547), pues este monarca estableció un moderno plan para organizar sus posesiones y las obras que tenían lugar en torno a ellos, a través de la institución Office of the King's Works, comprando, construyendo y reformando más de 60 palacios (Thurley, 1993 y 2017). Tras su muerte, comenzó el declinar de los Sitios Reales ingleses, con numerosos proyectos inacabados y con dificultad para mantener los ya existentes. De hecho, no encontramos en Inglaterra

12. En este sentido, los estudios de Monique Chatenet han contribuido enormemente a comprender este sistema, en especial su monografía Chatenet, M. (2002). La cour de France an XVIe siècle: vie sociale et architecture. París: Picard, así como sus numerosos trabajos sobre los castillos del Loira. 
JOSÉ ELOY HORTAL MUÑOZ

LOS SITIOS REALES COMO ELEMENTOS CLAVE DE LAS MONARQUÍAS EUROPEAS

DE LA EDAD MODERNA: UNA APROXIMACIÓN

ningún edificio comparable al Buen Retiro (Brown y Elliott, 2016), Versalles ${ }^{13}$ o Schönbrunn (Iby y Koller, 2000), que se erigieron durante el siglo XVII para reemplazar o complementar el Sitio Real principal en otras monarquías ${ }^{14}$.

En el caso de la Monarquía Hispana, como es bien sabido, Madrid fue la capital tras 1561 y la antigua fortaleza morisca del Real Alcázar se convirtió en el Sitio Real principal hasta la llegada de los Borbones en el siglo XVIII (Gérard, 1984; Barbeito, 1992; Checa, 1994).

La segunda categoría la constituían los Sitios Reales secundarios, los cuáles estaban localizados en su mayor parte fuera de la capital, y solían alojar a los monarcas durante sus cambios estacionales de aposento. Los reyes hispanos se movían entre El Pardo, Aranjuez, Valsaín y El Escorial; mientras, los reyes franceses viajaban a Villars-Cotterêts, Chambord, Fontainebleau, Versalles durante sus primeros años de existencia, y Compiègne; por su parte, en el Sacro Imperio los emperadores se movían entre Prater, Neugebäude, Luxemburg, Favorita y Ebersdorf ${ }^{15}$; finalmente, en Inglaterra, desde la Edad Media durante los meses de verano (agosto a octubre), los reyes solían alternar su estancia entre lugares de caza y de veraneo, mientras que en los meses de invierno se alojaban en los diversos Sitios Reales que se encontraban en la cercanía de la capital, dependiendo de las preferencias de cada monarca. En un principio se pactaba el itinerario con los grandes nobles, pero Enrique VII decidió marcar un itinerario fijo con Windsor al inicio de la temporada de invierno y, posteriormente, la corte marcharía a Westminster, Sheen y Greenwich para volver a Windsor.

Finalmente, la tercera categoría correspondía a los Sitios Reales de otros miembros de la familia real diferentes al Soberano. Como ya hemos indicado, cada miembro de la familia real tenía su propia Casa, y cuándo recibían oficios o puestos fuera de la Corte cómo gobernadores, gobernadores generales, virreyes u otras formas de

13. El Centre de recherche du château de Versailles tiene en la actualidad unos excelentes programas de investigación sobre éste Château, así como de otros en Francia o en otros lugares de Europa. Se pueden ver sus diversas publicaciones y actividades en http://chateauversaillesrecherche.fr/.

14. Es lo que Gérard Sabatier denominó palais d'État, que fueron apareciendo desde finales del siglo XVI en toda Europa hasta que a finales del siglo XVII existían en prácticamente todos los reinos, siendo una de sus principales funciones la legitimación dinástica (Sabatier, G. (2003). Le palais d'État en Europe, de la Renaissance au Grand Siècle. En M.-F. Auzépy y J. Cornette (dirs.), Palais et pouvoir. De Constantinople à Versailles. Saint-Denis: Presses Universitaires de Vincennes, 81-107).

15. Para 1692, el itinerario aparece descrito en Jeitler. Hunt, Amusement and Representation, 277, «A regular seasonal rotation of the court's presence in the Hofburg and the three nearby castles can be found out for the year 1692; toward the end of April or Early May the court moved to Laxenburg, on 2 June to Favorita and on 4 September for a few weeks to Ebersdorf, before returning to the Hofburg».

Ediciones Universidad de Salamanca / @®@@ Stud. his., H. ${ }^{a}$ mod., 42, n. 2 (2020), pp. 197-217 
poder delegado, se trasladaban a dichos lugares. Este fue el caso de los archiduques Habsburgo en Graz o Innsbruck, los diversos miembros de la Casa Real hispana en Nápoles, Bruselas, Palermo, Zaragoza, Lima u otras Cortes de la Monarquía, o los diferentes virreyes que los reyes ingleses enviaron a Escocia o Irlanda.

Igualmente, nos encontramos con otros Sitios Reales que fueron designados para otros miembros de la familia real en las capitales o en localidades cercanas. Esto no fue muy común en la Monarquía Hispana, pues únicamente podemos señalar el caso del Palacio de la Zarzuela, que fue mandado edificar por el Cardenal Infante, pero que pasaría a su hermano Felipe IV cuando falleció en Flandes (Toajas Roger, 2007). La situación fue diferente en Francia (donde la famille royale tenía diversos palacios), Austria (donde Ameliemburg fue designado para los archiduques y las emperatrices viudas), e Inglaterra (Somerset House se reservaba para reinas y St. James's Palace para los príncipes de Gales antes de ser designado Sitio Real principal).

Gracias a ello, la disposición espacial de dichos Sitios Reales se caracteriza hoy en día por una morfología similar en todos los rincones de Europa (Versalles, Tsarskoie Selò, Hampton Court, Aranjuez,..), pues todos ellos comparten algunas características que daban forma a una identidad específica. Sus asentamientos eran diferentes a otras comunidades rurales o urbanas europeas, debido a su especial relación con el Soberano y la dinastía reinante, y al hecho de que sus sociedades fueron creadas a través de experimentos sociales de motivación urbana, económica, cultural y, en ocasiones, también religiosa.

En efecto, los Sitios Reales tuvieron también consecuencias notables en las transformaciones sociales europeas de los siglos XV al XVIII ${ }^{16}$. En este sentido, los Sitios Reales fueron espacios de interacción cortesana, estructurados por la Etiqueta, el Ceremonial y las normas de conversación. Las «buenas maneras» que tenían cabida en esos lugares, expresaban las cualidades morales de la aristocracia cortesana, al tiempo que la magnificencia y la belleza arquitectónica de los palacios expresaban la armonía y el gobierno virtuoso de los soberanos (Versteegen, 2015).

Por otro lado, el carácter innovador de estos lugares provocó el incremento de la diversificación de la economía y de la población con una profesión concreta, así como la emergencia de centros urbanos. Por lo tanto, se convirtieron en espacios extremadamente interesantes para estudiar como la sociedad moderna se desarrolló y coexistió con estructuras sociales cortesanas.

Los palacios reales eran más que meras residencias de los soberanos y sus cortes y casas reales, pues eran microsociedades que estructuraban políticamente las monarquías y reinos a través de las redes clientelares. Considerando a estos

16. Sobre el carácter particular de la sociedad de la Edad Moderna, Schlögl, R. (2014). Anwesende und Abwesende. Grundriss für eine Gesellschafts-Geschichte der früben Neuzeit. Constanza: Konstanz University Press. 
Sitios Reales como espacios compuestos y centros de innovación, la imagen que podemos ver de los mismos es mucho más compleja y dinámica de lo que ha venido siendo considerado hasta ahora. Los Sitios Reales crearon demandas específicas de productos y servicios que llevaron a innovaciones económicas, artísticas y científicas cuyas consecuencias pueden ser vistas en numerosos aspectos, como la explotación agrícola de las tierras circundantes, la introducción de nuevas técnicas en la administración de los bosques reales, la arquitectura y el diseño de los jardines, la creación de fábricas para productos de lujo como los gobelinos o la porcelana, o la construcción de palacios para los príncipes y nobles, incluyendo colecciones de pinturas, esculturas, muebles, cámaras de las maravillas, instrumentos de música,...

Todo ello atrajo a artistas, autores, emprendedores, arquitectos, paisajistas, músicos, inventores, académicos, científicos o abogados, que estaban buscando una oportunidad en la Corte ${ }^{17}$. Junto a los servidores reales formaron una población heterogénea que conformaría el núcleo de centros urbanos que se irían desarrollando al albur de estos lugares, con características propias y diversas a las de su entorno. Del mismo modo, la reorganización del territorio causaría una profunda transformación del paisaje y cambió las relaciones entre la Corte y los municipios afectados por su extensión.

Al tiempo que todo esto sucedía, se crearon nuevos espacios de sociabilidad, pues también debemos tener en cuenta como durante el siglo XVII se produjo el paulatino traslado de la fiesta cortesana al interior de los palacios reales y, por tanto, a la vista de los cortesanos, pero fuera del alcance del pueblo (Alewyn, 1964; Baillie, 1967). De este modo, se confirmaba el ocultamiento del monarca y de la apreciación de su Majestad por parte de sus súbditos. Para ello, fue necesario que se dieran varios pasos.

En primer lugar, que algunas monarquías destinaran cuerpos de guarda preexistentes, o crearan nuevos, para proteger los Sitios Reales donde se iban a llevar a cabo esas fiestas y ceremonias, separando así esos espacios físicamente del resto de la sociedad.

Paradigmático fue sin duda el caso de Francia, reino que desde la muerte de Enrique II y el inicio de las guerras de religión estaba sufriendo una inestable situación política, lo que tendría su reflejo evidente en las unidades de guarda. Así, se incrementaría notablemente al alza el número y la cantidad de componentes de esas unidades, con el fin de actuar como apaciguadoras de la situación, además de ir

17. Por ejemplo, en ANF, O1, 2388, s. f., encontramos un listado de «Officiers servant Sa Maiestè pour l'entreteniement de ses maisons et chasteaux cy apres detaillés» de 1664, donde aparecen en el apartado «Genre de lettres estrangere» a 13 astrólogos, físicos, médicos,... todos ellos extranjeros, financiados por el monarca francés para que trabajaran en sus Sitios Reales. En 1665 serían ya 17. 
apareciendo una división entre las unidades que no había existido hasta el momento: las guardas «du dedans et $d u$ dehors $d u$ Louvre» ${ }^{18}$.

Este conglomerado serviría de modelo a numerosos reinos europeos, entre ellos Inglaterra, con la creación de la Royal Military Household, o el Sacro Imperio, con la Schlossgarde derivada de los guardabosques del exterior de los palacios (Hortal Muñoz, 2013: cap. 2). Sin embargo, el modelo no pudo ser adaptado adecuadamente a la guarda real de la Monarquía Hispana en el siglo XVII, que estaría más cerca de ser un cuerpo de parada y ceremonial que una verdadera fuerza militar o de vigilancia de dichos Sitios Reales (Hortal Muñoz, 2011), y no se crearían cuerpos específicos para dichas tareas hasta el siglo XVIII (Martínez y Pi Corrales, 2010). Pese a ello, durante toda la Edad Moderna nos encontramos con guardas de a pie o de a caballo, así como porteros, en Sitios Reales como El Pardo, Aranjuez, La Casa de Campo, El Escorial o Soto de Roma ${ }^{19}$, lo que nos indica que ya eran espacios claramente diferenciados de su entorno, aunque con mayor libertad de paso.

El siguiente paso en dicho proceso, fue la creación de nuevas infraestructuras en los Sitios Reales diseñadas especialmente para las celebraciones cortesanas, destacando los escenarios ilusionistas que fomentaban las representaciones interiores y los teatros de corte permanentes, como el Coliseo del Buen Retiro o el teatro del Pardo en la Monarquía Hispana (Ferrer, 1995; Flórez, 1998), Banqueting House en Londres, o los teatros cortesanos del palacio de Versalles o del de Schönbrunn; dicha moda se extendería al resto de Europa, en especial a partir del siglo XVIII, con ejemplos tan notables como el teatro cortesano del Palacio Real de Nápoles o el de Sans Soucci entre muchos otros.

En resumen, mientras los temas de la fiesta cortesana renacentista suponían la contemplación de la armonía cósmica y su reflejo en la sociedad, en el Barroco se representaría al monarca como génesis de esa armonía, en una esfera diferente a la de sus súbditos y completamente fuera del alcance de estos, excepto para aquellos moradores de los Sitios Reales que tenían el privilegio de asistir a dichos eventos (Strong, 1988: 171-172).

Sin duda, el siglo XVII fue esencial en la configuración de los espacios cortesanos, así como en lo referente a la articulación espacial interna de estos lugares en relación al ceremonial y a las Casas Reales. En este contexto, diversas cortes llevaron a cabo el proceso de fijación de sus Etiquetas y, por ejemplo, en Francia, Teodoro Godefroy sistematizó, en 1619, la colección de escrituras ceremoniales francesas que

18. Sobre la evolución de las guardas reales francesas y una recopilación bibliográfica, Hortal Muñoz, J. E. (2013). Las guardas reales de los Austrias hispanos. Madrid: Polifemo, 63-80.

19. Ver biografías y casos en Martínez Millán, J. y Hortal Muñoz, J. E. (dirs.). (2015). La Corte de Felipe IV (1621-65). Reconfiguración de la Monarquía Católica: Madrid: Polifemo, en el CD del tomo II. 
Denis II, historiador francés, alargó y volvió a publicar en Paris en 1649 como Le ceremonial français. Otro ejemplo lo constituye Florencia, donde no hubo diario de Etiquetas antes de 1589, pero para el siglo XVII nos encontramos con 33. En el caso madrileño, se produciría la publicación de las famosas Etiquetas Generales de Palacio en 1651, así como la codificación en otras cortes de la Monarquía como Nápole ${ }^{20}$ o Bruselas (Hortal, Pirlet y Espíldora, 2018: 9-59, especialmente 34). Sin duda, estas modificaciones legislativas constataron la relevancia in crescendo que los Sitios Reales tendrían en los principales rituales dinásticos y representativos de las monarquías, -caso de funerales, bautizos...

Finalmente, conviene indicar que estos lugares fueron acumulando numerosos tesoros arquitectónicos y artísticos, al tiempo que se construyeron allí librerías, boticas, archivos, laboratorios, gabinetes científicos y centros manufactureros o fábricas, esto último en especial a partir del siglo XVIII. Por lo tanto, los Sitios Reales pueden ser considerados como centros de cultura, ciencia e innovación que contribuyeron al progreso y desarrollo de la economía y sociedad europea y de otros territorios en el mundo, convirtiéndose también en centros de intercambio cultural en todos sus aspectos.

En este sentido, los Sitios Reales fueron centros dinámicos de una cultura europea común, pues los cortesanos que llegaban a dichos lugares desde otras cortes foráneas sabían los códigos dominantes de comportamiento y las reglas de la práctica política, así como la Etiqueta, a pesar de algunas mínimas diferencias locales o lingüísticas.

\section{NUEVAS PERSPECTIVAS DE ESTUDIO}

En virtud de todo ello, consideramos que es necesario estudiar los Sitios Reales desde nuevas perspectivas, con el fin de poder comprender la verdadera relevancia de dichos lugares, tal y cómo se viene realizando en los últimos años ${ }^{21}$.

20. Gracias al progetto cerimoniali (https://www.progettocerimoniali.org/), hoy en día la Corte de Nápoles es la mejor estudiada en este aspecto de la Monarquía Hispana, gracias a los cuatro monumentales volúmenes que abarcan las diversas fases históricas de los territorios napolitanos.

21. Para el caso de la Monarquía Hispana, por ejemplo, se ha ido evolucionando desde los trabajos clásicos como Checa Cremades, F. y Morán Turina, J. M. (1986). Las Casas del Rey. Casas de Campo, Cazaderos y Jardines. Siglos XVI y XVII. Madrid: El Viso, o Sancho Gaspar, J. L. (1995). La arquitectura de los Sitios Reales: catálogo bistórico de los palacios, jardines y patronatos reales del Patrimonio Nacional. Madrid: Patrimonio Nacional y Fundación Tabacalera, a los más recientes, caso de los capítulos dedicados a ello en Martínez Millán y Hortal Muñoz, La Corte de Felipe IV, o algunos de los trabajos recopilados en Camarero Bullón, C. y Labrador Arroyo, F. (dirs.) (2017). La extensión de la corte: los Sitios Reales. Madrid: UAM. Para el siglo XVIII, ver dos monográficos de revista; el dirigido por Cirillo, G. y Grimaldi, A. (2017). The Europe of "decentralised courts». Palaces and Royal Sites: the construction of the political image of the Bourbons of Italy and Spain. Cheiron, número monográfico 2; y el

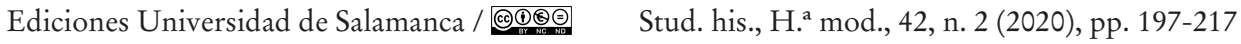


JOSÉ ELOY HORTAL MUÑOZ

LOS SITIOS REALES COMO ELEMENTOS CLAVE DE LAS MONARQUÍAS EUROPEAS

DE LA EDAD MODERNA: UNA APROXIMACIÓN

Para ello, consideramos que se debe imbricar su estudio dentro de algunas de las metodologías en Humanidades más usadas en la actualidad, en especial vinculadas al estudio del espacio. Este el caso del denominado spatial turn, que nos puede ayudar a comprender mejor el espacio cortesano a través de los estudios que se están llevando a cabo desde el cambio de siglo, siguiendo una tendencia que inició el historiador marxista Henri Lefebvre en la década de los 80 del siglo $\mathrm{XX}^{22}$. Según estos estudios, los historiadores deben prestar cada vez una mayor atención al uso y concepción del espacio, no como algo inerte sino estructural, y destacando la necesidad de situarlo en el centro de las concepciones teóricas (Crang, 2009; Kingston, 2010), en especial para la Edad Moderna (Stock, 2015). Sin duda, el espacio, entendido como una construcción, sirve para comprender cómo se ha dominado y organizado a la sociedad, por lo que conviene estudiar su papel en los procesos históricos y conflictos ${ }^{23}$.

Por supuesto, el espacio jugó también un papel fundamental en la configuración del sistema cortesano, aunque hasta hace unos años únicamente se utilizaba dicha categoría para estudiar palacios y ciudades donde moraba la Corte con frecuencia, y en especial el impacto que esta producía en las ciudades que la acogían ${ }^{24}$. Sin embargo, hoy en día, se han comenzado a estudiar las interacciones políticas, prácticas ceremoniales, símbolos culturales y estructuras institucionales de la Corte ${ }^{25}$. En este sentido, resulta muy interesante la Actor-Network Theory (ATN) iniciada

dirigido por Martínez, J., González, N.; Valido-Viegas, F. M. (2018). Casas y Sitios Reales en las monarquias de España y Portugal. Libros de la Corte, 17. Estos últimos trabajos, están ayudando a identificar el relevante papel que jugaron los Sitios Reales en el desarrollo político, social, económico y cultural de la Monarquía Hispana en su conjunto.

22. En los últimos años, ha ido aumentando progresivamente la atención al Spatial Turn en numerosas disciplinas, tal y como se explica en Warf, B. y Arias, S. (dirs.). (2009). The spatial turn: interdisciplinary perspectives. Londres: Routledge, especialmente la introducción.

23. En especial, Bravo, P. y d'Amico, J. C. (dirs.). (2017). Territoires, lieux et espaces de la révolte. XIVe-XVIIIe siècles. Dijon: Editions Universitaires de Dijon. En este libro, podemos encontrar bibliografía sobre el Spatial Turn en p. 23, n. 2. Por su parte, un estado de la cuestión sobre el papel del espacio en la historia social y cultural hasta 2014 en Williamson, F. (2014). The Spatial Turn of Social and Cultural History: A Review of the Current Field, European History Quarterly, 44(4), 703-717.

24. En la actualidad, hay numerosas investigaciones referentes al impacto de la Corte en la ciudad, tal y cómo nos muestra Courbon, L. y Menjot, D. (2015). La cour et la villa dans l'Europe du Moyen âge et des Temps Modernes. Studies in European Urban History (1100-1800), 35, o el ya citado Bove, Gaude-Ferragu y Michon, Paris, ville de cour. Pionero en este campo fue Spielman, J. P. (1993). The City and the Crown: Vienna and the Imperial Court 1600-1740. West Lafayette: Purdue University Press.

25. El pistoletazo de salida sobre la aplicación del Spatial Turn en los estudios sobre la Corte lo puede constituir la obra de Fantoni, M., Gorse, G. y Smuts, M. (eds.). (2009). The Politics of Space: European Courts ca. 1500-1750. Roma: Bulzoni, donde se enuncian sus parámetros en la introducción. 
por Bruno Latour y Steve Woolgar en los 80 con su libro Laboratory life, y que posteriormente desarrollaron otros autores como John Law o John Hassard. Dicha teoría estudia la interacción de la gente con el territorio, lo que constituye un punto clave para el estudio de espacios tan peculiares como los Sitios Reales, donde la inmensa mayoría de las personas que allí vivían trabajaba para el monarca. Desde este punto de vista, el espacio no debe ser considerado como una característica fija, sino que se debe estudiar según el modo en el que se ocupe. Por lo tanto, no se debe separar el estudio del espacio (space en inglés y espace en francés) del de sitio o lugar (place o lieu), aunque el primer concepto es más abstracto que el segundo (Dillon, 2010: 1-17), pues los dos están construidos a través de la percepción.

En virtud de ello, aunque en la definición clásica de Corte siempre se nos ha hablado únicamente del lugar donde moraba el rey y su séquito, debemos tener en cuenta, no solo, el punto de vista físico, sino también el simbólico. Por lo tanto, la Corte comprendería también aquellos lugares en donde estuviera presente el monarca, tanto en su presencia física como metafórica; circunstancia que cobró especial relevancia en las monarquías europeas modernas a través de los Sitios Reales.

Del mismo modo, y vinculada al Spatial Turn, el uso de la historia transregional también puede resultar muy fructífero en este sentido ${ }^{26}$. Hasta hace unos años, las fronteras en la Edad Moderna eran vistas como una extrapolación de las actuales, así como se ligaban a cuestiones relacionadas con el Estado y su construcción, ya que no existía un marco teórico sobre las construcciones espaciales del periodo como sí suponía el Estado para momentos posteriores. Sin embargo, la historia transregional tiene como clave que las fronteras de la Edad Moderna no necesariamente reflejaban la organización del territorio según separaciones singulares, si no que eran mucho más variables. Por lo tanto, no habría que predefinir la escala espacial de la investigación para poder centrarse más en aquellos elementos que se movían, incluidos y, sobre todo, grupos de personas que trascendían las fronteras de la época, así como elementos transculturales.

Los Sitios Reales entrarían claramente en esta categoría, pues fueron centros culturales y económicos dinámicos que trascendían las fronteras de las monarquías multinacionales del momento ${ }^{27}$, y que ayudaron a crear una verdadera cultura europea, así como a establecer contactos con otras culturas en América, África o

26. Metodología explicada por un grupo de investigación de la Universidad de Lovaina (Bélgica) en Soen, V. et alii (2017). How to do Transregional History: A Concept, Method and Tool for Early Modern Border Research, Journal of Early Modern History, 21, 343-364. Los principales avances de dicho grupo sobre estas cuestiones se pueden consultar en su web http://transregionalhistory.eu/.

27. Entendido el concepto de nación tal y cómo se explica en Herzog, T. (2003). Defining Nations: Inmigrants and Citizens in Early Modern Spain and Spanish America. New Haven/ Londres: Yale University Press. 
Asia, en el caso de monarquías como la hispana o la portuguesa. De este modo, fijaron los estándares y tendencias en arte, moda y conocimiento, constituyendo una red diversa y global que ayudaba a que el poder real fuera visible y efectivo. Del mismo modo, y como ya hemos señalado anteriormente, los habitantes vinculados a los Sitios Reales compartían una serie de códigos comunes que les servían para manejarse en esos ámbitos a lo largo de toda Europa. Por lo tanto, no resulta extraño que, por ejemplo, el eminente músico Georg Friedrich Händel pudiera componer y residir tanto en cortes católicas como protestantes.

Pero, sin duda, y en especial, los Estudios de la Corte que se vienen desarrollando durante las últimas décadas están llevando el estudio de los Sitios Reales a una nueva dimensión ${ }^{28}$. En virtud de dichos estudios, aunque la definición clásica de Corte nos hablaba de que estaba compuesta por tres elementos (Casa Real, Consejos y Tribunales y cortesanos), dejaba fuera uno muy importante como fueron los Sitios Reales, en especial desde el siglo XVII, tal y como se viene investigando en los últimos años y derivados de la propia evolución de las diversas monarquías (Hortal y Versteegen, 2016: cap. 1).

Por lo tanto, estamos convencidos de que el estudio de los Sitios Reales a través de nuevas claves metodológicas seguirá dando relevantes frutos en los próximos años.

\section{BIBLIOGRAFÍA}

Alewyn, R. (1964). L'Univers du baroque, les fêtes baroques. Ginebra: Gonthier.

Allsen, T. T. (2006). The Royal Hunt in Eurasian History. Philadelphia: University of Penssilvania Press.

Anthenaise, C. d. y Chatenet, M. (eds.). (2007). Chasses princières dans l'Europe de la Renaissance. Arles: Actes Sud.

Baillie, H. M. (1967). Etiquette and the planning of state apartments in baroque palaces, Archaeologia or miscellanous tracts relating to antiquity, 101, 167-199.

Barbeito, J. M. (1992). El Alcázar de Madrid. Madrid: COAM.

Barbiche, B. (1984). Henri IV et la surintendance des bâtiments. Bulletin Monumental, 142(1), 19-39.

Bove, B., Gaude-Ferragu, M. y Michon, C. (dirs.) (2017). Paris, ville de cour (XIIIe-XVIIIe siècle). Rennes: Presses Universitaires de Rennes.

Bravo, P. y d'Amico, J. C. (dirs.). (2017). Territoires, lieux et espaces de la révolte. XIVeXVIIIe siècles. Dijon: Editions Universitaires de Dijon.

28. Una puesta al día de la investigación sobre la Corte se puede ver en Duindam, J. (2015). Rulers and Courts. En H. Scott (dir.) (2015). The Oxford Handbook of Early Modern European History, 1350-1750: Cultures and Power. Cambridge: Cambridge University Press, II, 440-477.

Ediciones Universidad de Salamanca / @®@@ Stud. his., H. ${ }^{a}$ mod., 42, n. 2 (2020), pp. 197-217 
JOSÉ ELOY HORTAL MUÑOZ

LOS SITIOS REALES COMO ELEMENTOS CLAVE DE LAS MONARQUÍAS EUROPEAS

DE LA EDAD MODERNA: UNA APROXIMACIÓN

Brown, J. y Elliott, J. H. (2016). Un palacio para el rey: el Buen Retiro y la corte de Felipe IV. Madrid: Taurus (reedición de la original de 1983).

Brunner, O. (2010). La «casa grande» y la «Oeconomica» de la vieja Europa. Prismas: revista de historia intelectual, 14, 117-136.

Bürger, S. (2009). Das wettinische Landeswerkmeisteramt - Sonderweg und Potential des obersächischen Bauwesens um 1500. En S. Bürger y B. Klein (eds.), Werkmeister der Spätgotik. Position und Rolle der Architekten im Bawwesen des 14. bis 16. Jabrbunderts (pp. 59-65). Darmstadt: Wissenschaftliche Buchgesellschaft.

Camarero Bullón, C. y Labrador Arroyo, F. (dirs.) (2017). La extensión de la corte: los Sitios Reales. Madrid: UAM.

Chapelot, J. (2001). Charles V maître d'ouvrage: à propos de la construction du donjon de Vincennes et de quelques chantiers contemporains. En O. Chapelot (ed.), Du Project au chantier. Maîtres d'ouvrage et maîtres d'oeuvre aux XIVe-XVIe siècles (pp. 339-403). París: Editions des Hautes Etudes en Sciences Sociales.

Chatenet, M. (2002). La cour de France au XVIe siècle: vie sociale et architecture. París: Picard.

Checa Cremades, F. (dir.) (1994). El Real Alcázar de Madrid. Dos siglos de arquitectura y coleccionismo en la corte de los Reyes de España. Madrid: Nerea.

Cirillo, G. y Grimaldi, A. (2017). The Europe of «decentralised courts». Palaces and Royal Sites: the construction of the political image of the Bourbons of Italy and Spain. Cheiron, número monográfico 2.

Colvin, H. M. (1963-1982). The history of the king's Works. Londres: H.M. Stationery Off. 6 vols.

Courbon, L. y Menjot, D. (2015). La cour et la villa dans l'Europe du Moyen âge et des Temps Modernes. Studies in European Urban History (1100-1800), 35.

Crang, M. (2009). Spaces in theory, spaces in history and spatial historiographies. En Beat A. Kümin (ed.). Political space in preindustrial Europe (pp. 249-265). Farnham: Ashgate.

Díaz González, F. J. (2002). La Real Junta de Obras y Bosques en la época de los Austrias. Madrid: Dykinson.

Dillon, J. (2010). The language of space in court performance, 1440-1625. Cambridge: Cambridge University Press.

Domínguez Casas, R. (1993). Arte y etiqueta de los Reyes Católicos. Artistas, residencias, jardines y bosques. Madrid: Alpuerto.

Duindam, J. (2003). Viena and Versailles. The Courts of Europe's Dynastic Rivals, 15501780. Cambridge: Cambridge University Press.

Duindam, J. (2015). Rulers and Courts. En H. Scott (dir.). (2015). The Oxford Handbook of Early Modern European History, 1350-1750: Cultures and Power (II, pp. 440-477). Cambridge: Cambridge University Press.

Engel, E. y Lambrecht, K. (1995). Hauptstadt-Residenz-Residenzstadt-Metropole-zentraler Ort. Probleme ihrer Definition und Charakterisierung. En E. Engel, K. Lambrecht y H. Nogossek (eds.). Metropolen im Wandel. Zentralität in Ostmitteleuropa an der

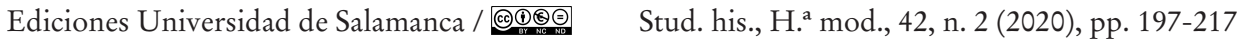


JOSÉ ELOY HORTAL MUÑOZ

LOS SITIOS REALES COMO ELEMENTOS CLAVE DE LAS MONARQUÍAS EUROPEAS

DE LA EDAD MODERNA: UNA APROXIMACIÓN

Wende vom Mittelalter zur Neuzeit. Berlín: De Gruyter Akademie Forschung (= Forschungen zur Geschichte und Kultur des ostlichen Mitteleuropa).

Fantoni, M., Gorse, G. y Smuts, M. (eds.). (2009). The Politics of Space: European Courts ca. 1500-1750. Roma: Bulzoni.

Ferrer Valls, T. (1995). Teatros cortesanos anteriores a la construcción del Coliseo del Buen Retiro. Quaderns de filologia. Estudis literaris, 1, 355-372.

Flórez Asensio, M. ${ }^{a}$ A. (1998). El Coliseo del Buen Retiro en el siglo XVII: teatro público y cortesano, Anales de Historia del Arte, 8, 171-195.

Frigo D. (1985). Il Padre di famiglia. Governo della Casa e governo civile nella tradizione dell'economica tra Cinque e Seicento. Roma: Bulzoni.

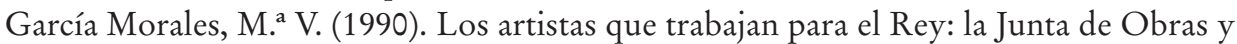
Bosques. Espacio, Tiempo y Forma. Historia del Arte, 3, 123-136.

Gérard, V. (1984). De castillo a palacio. El Alcázar de Madrid en el siglo XVI. Madrid: Xarait.

Harvey, J. H. (1941). The Medieval Office of Works. Journal of the British Archaeological Association, 6, 20-87.

Hausenblasova, J. (2017). A New Monarch and a New System of Residences: Ferdinand I Habsburg as the Founder of the Network of Main and Occasional Residences in the Habsburg Empire. En S. Dobalova e I. P. Muchka (eds.), Looking for Leisure. Court Residences and their Satellites, 1400-1700 (pp. 46-61). Praga: Palatium.

Herzog, T. (2003). Defining Nations: Inmigrants and Citizens in Early Modern Spain and Spanish America. New Haven/Londres: Yale University Press.

Hortal Muñoz, J. E. (2011). Las guardias de los Austrias, ¿cuerpo militar o de parada?. En S. Granda Lorenzo, L. Martínez Peñas y M. Fernández Rodríguez (coords.), Perspectivas jurídicas e institucionales sobre guerra y ejército en la Monarquía Hispánica (pp. 119-151). Madrid: Dykinson.

Hortal Muñoz, J. E. (2013). Las guardas reales de los Austrias hispanos. Madrid: Polifemo.

Hortal Muñoz, J. E. (2014). La integración de los Sitios Reales en el sistema de Corte durante el reinado de Felipe IV. Libros de la Corte, 8, 27-47.

Hortal Muñoz, J. E. (2017). La unión de la Corte, la Casa y el territorio en la Monarquía Hispana de los siglos XVI y XVII: las guardas reales y los Sitios Reales. Revista Escuela de Historia, 16(1).

Hortal Muñoz, J. E. (dir.) y Pirlet, P.-F. y Espíldora García, Á. (eds.). (2018). El ceremonial en la Corte de Bruselas del siglo XVII. Los manuscritos de Francisco Alonso Lozano, Bruselas: Commission Royale d'Histoire / Koninklijke Commissie voor Geschiedenis.

Hortal Muñoz, J. E. y Versteegen, G. (2016). Las ideas politicas y sociales en la Edad Moderna. Madrid: Síntesis.

Iby, E. y Koller, A. (2000). Schönbrun. Viena: Verlag Christian Brandstätter.

Jacquier, É. (2001). La politique castrale des Valois de Bourgogne dans les bailliages méridionaux de leur duché. En Chapelot, Du project au chantier, 143-162.

Jeitler, M. (2017). Hunt, Amusement and Representation: The Viennese Hofburg and Its 'Satellites' in the Seventeenth Century. En Dobalova y Muchka, Looking for Leisure, 274-280.

Ediciones Universidad de Salamanca / @®@@ Stud. his., H. ${ }^{a}$ mod., 42, n. 2 (2020), pp. 197-217 
JOSÉ ELOY HORTAL MUÑOZ

LOS SITIOS REALES COMO ELEMENTOS CLAVE DE LAS MONARQUÍAS EUROPEAS

DE LA EDAD MODERNA: UNA APROXIMACIÓN

Karner. H. (dir.). (2014). Die Wiener Hofburg 1521-1705. Viena: Denkschriften Der Philosophisch-historischen Klasse.

Kingston, R. (2010). Mind Over Matter? History and the Spatial Turn. Cultural and Social History, 7(1), 111-121.

Kuper, H. (1972). The Language of Sites in the Politics of Space, American Anthropologis, 74, 411-425.

Kurdiovsky, R. et alii (2015). Legitimacy through History and Architecture: The Vienna Hofburg as Dynastic Hub and Seat of Government between Tradition and Innovation. Court Historian, 20(2), 109-136.

Lambertini R. (1984). Per una storia dell'oeconomica tra alto e basso Medioevo. Cheiron, $4,45-74$.

Lardin, P. (1999). Des hommes de terrain : les maîtres du roi en Normandie orientale à la fin du Moyen Âge. En Les serviteurs de l'état au Moyen Âge (XXIXe Congrès de la S.H.M.E.S. Pau, mayo 1998) (pp. 133-179], París: Publications de la Sorbonne.

Maroteaux, V. (2000). Versailles. Le Roi et son Domaine. París: Editions Picard.

Martínez Millán, J. (2017). La descomposición del sistema cortesano: la supresión de la Junta de Obras y Bosques. En P. Broggio, L. Guarnieri Calò Carducci y M. Merluzzi (eds.), Europa e America allo specchio. Studiper Francesca Cantù. Roma: Viella, 159-186.

Martínez Millán, J., González Heras, Natalia; Valido-Viegas De Paula-Soares, Felipa M. ${ }^{a}$ (2018). Casas y Sitios Reales en las monarquias de España y Portugal. Libros de la Corte, 17.

Martínez Millán, J. y Hortal Muñoz, J. E. (dirs.). (2015). La Corte de Felipe IV (1621-65). Reconfiguración de la Monarquía Católica: Madrid: Polifemo, 2 tomos.

Martínez Ruíz, E. y Pi Corrales, M. de P. (2010). Protección y seguridad en los Sitios Reales. Desde la Ilustración al liberalismo. Alicante: Universidad.

Merlotti, A. (2016). Cortes, capitales y residencias en la Europa católica de los siglos XVII y XVIII. Puntos de reflexión sobre un problema político. En J. L. Sancho Gaspar y J. Ortega Vidal, Una Corte para el Rey: Carlos III y los Sitios Reales, catálogo de exposición (pp. 22-35). Madrid: Comunidad Autónoma de Madrid, servicio de documentación y publicaciones.

Merlotti, A. (dir.). (2017). Le Cacce Reali nell'Europa dei Principi. Turín: Leo S. Olschki.

Nan Rosenfeld, M. (1977). The Royal Building Administration in France from Charles V to Louis XIV. En S. Kostof (ed.), The Architect. Chapters in the History of the Profession (pp. 161-179). Nueva York: Oxford University Press.

Newman, C. (1998). Reflections on the making of a 'royal site' in early Ireland, World Archaeology, 30(1), 127-141. https://doi.org/10.1080/00438243.1998.9980401

Poisson, J.-M. (2001). La maîtrise d'oeuvre dans les chantiers de construction des châteaux du comté de Savoie au XIVe siècle. En Chapelot, Du project au chantier, 163-175.

Sabatier, G. (2003). Le palais d'État en Europe, de la Renaissance au Grand Siècle. En M.-F. Auzépy y J. Cornette (dirs.), Palais et pouvoir. De Constantinople à Versailles (pp. 81-107). Saint-Denis: Presses Universitaires de Vincennes.

Ediciones Universidad de Salamanca / @®@@ Stud. his., H. ${ }^{a}$ mod., 42, n. 2 (2020), pp. 197-217 
JOSÉ ELOY HORTAL MUÑOZ

LOS SITIOS REALES COMO ELEMENTOS CLAVE DE LAS MONARQUÍAS EUROPEAS

DE LA EDAD MODERNA: UNA APROXIMACIÓN

Sancho Gaspar, J. L. (1995). La arquitectura de los Sitios Reales: catálogo bistórico de los palacios, jardines y patronatos reales del Patrimonio Nacional. Madrid: Patrimonio Nacional y Fundación Tabacalera.

Sancho Gaspar, J. L. y Martínez Leiva, G. (2003). ¿Dónde está el rey? El ritmo estacional de la corte española y la decoración de los Sitios Reales (1650-1700). En Cortes del Barroco. De Bernini y Velázquez a Luca Giordano. Catálogo de la exposición (pp. 85-98). Madrid: Sociedad Estatal para la Acción Cultural Exterior.

Sarmant, T. (2003). Les demeures du soleil: Louis XIV, Louvois et la surintendance des bâtiments du roi. Seyssel: Champ Vallon.

Schlögl, R. (2014). Anwesende und Abwesende. Grundriss für eine Gesellschafts-Geschichte der früben Neuzeit. Constanza: Konstanz University Press.

Soen, V. et alii (2017). How to do Transregional History: A Concept, Method and Tool for Early Modern Border Research, Journal of Early Modern History, 21, 343-364.

Spielman, J. P. (1993). The City and the Crown: Vienna and the Imperial Court 1600-1740. West Lafayette: Purdue University Press.

Stock, P. (ed.) (2015). The Uses of Space in Early Modern History. Nueva York: Palgrave Macmillan.

Strong, R. (1988). Arte y poder. Fiestas del Renacimiento, 1450-1650. Madrid: Alianza Editorial.

Toajas Roger, M. a de los Á. (2007). La heredad de la Zarzuela. Nuevos documentos de su historia, Anales de Historia del Arte, 17, 85-116.

Thurley, S. (1993). The Royal Palaces of Tudor England. Architecture and Court life 14601547. New Haven y Londres: Yale University Press.

Thurley, S. (2017). Houses of Power: The places that shaped the Tudor world. Londres: Bantam Press.

Velázquez Muñoz, J. (2011). El Gran Rey en movimiento. Banquetes y partetaš. Espacio, Tiempo y Forma. Serie II, Historia Antigua, 24, 161-188.

Vermeir, R., Raeymaekers, D. y Hortal Muñoz, J. E. (eds.). (2014). A Constellation of Courts. The households of Habsburg Europe, 1555-1665. Lovaina: Leuven University Press.

Versteegen, G. (2015). Corte y Estado en la historiografía liberal. Un cambio de paradigma. Madrid: Polifemo.

Warf, B. y Arias, S. (dirs.). (2009). The spatial turn: interdisciplinary perspectives. Londres: Routledge.

Williamson, F. (2014). The Spatial Turn of Social and Cultural History: A Review of the Current Field, European History Quarterly, 44(4), 703-717.

Ediciones Universidad de Salamanca / @@@ Stud. his., H. ${ }^{a}$ mod., 42, n. 2 (2020), pp. 197-217 
